\title{
Researchers create 1,000-robot swarm
}

Tiny troupe could shed light on collective behaviour in animals and humans.

\section{Mark Zastrow}

14 August 2014

\section{Robot swarm on the loose}

Tiny robots called Kilobots can form different shapes by collective action.

MICHAEL RUBINSTEINHARVARD UNIVERSITY

Scientists have created a swarm of over a thousand coin-sized robots that can assemble themselves into two-dimensional shapes by communicating with their neighbours.

At 1,024 members, this man-made flock — described in the 15 August issue of Science ${ }^{1}$ — is the largest yet to demonstrate collective behaviour. The self-organization techniques used by the tiny machines could aid the development of 'transformer' robots that reconfigure themselves, researchers say, and they might shed light on how complex swarms form in nature.

The puck-shaped robots, called Kilobots, cost roughly US $\$ 20$ each and are programmed with a simple set of rules and an image of the shape to be formed. To begin with, the robots are arranged in a tightly packed, arbitrary shape on a flat surface. Then four 'seed robots' are placed in a cluster next to the swarm, and the robots on the far side of the pack begin to inch around the edge of the formation towards the seeds, propelled by motors that make them vibrate like ringing mobile phones.

The robots communicate using infrared light, but they are only able to transmit and receive information with the robots nearest to them — so they cannot 'see' the whole collective. However, the seed robots act as the point of origin for a coordinate system; information on their position propagates outward through the swarm like fire signals across the peaks of a mountain range. This allows each bot to determine where it is and whether it is inside the shape programmed by researchers. Over a period of about 12 hours, the programmed configuration — such as the letter ' $K$ ' or a star — takes form, robot by robot.

\section{Shape-shifters}

Building and operating such a large swarm is an impressive engineering feat, says Mark Yim, a robotics engineer at the University of Pennsylvania in Philadelphia, who was not involved in the study. Previous demonstrations of swarming programmable robots involved 
roughly 50 units, he says.

This shape-shifting robot flock is analogous to ants that build bridges out of their own bodies, demonstrating modular behaviour that allows them to adapt quickly to their surroundings, says Michael Rubenstein, a computer scientist at Harvard University in Cambridge, Massachusetts, and the study's lead author.

He thinks that such behaviour could be useful in creating programmable matter: tiny robots the size of sand grains that could rearrange themselves into tools of any shape, such as a wrench. These future robots would act "like a three-dimensional printer, but instead of printing with plastic filament, you'd be printing with robots that can move themselves", says Rubenstein.

Even more futuristic applications of these shape-shifting techniques might be in reconfigurable robots — real-life Transformers. Yim has built a modular robot that can shift itself from a four-legged configuration to a snake-like chain, and says the new study could help to further refine such transformations. ${ }^{2}$

But the Kilobots are not foolproof. A slow bot can cause 'traffic jams' along the outer edges, and the shapes tend to look warped owing to the Kilobots' imprecise tracking and their tendency to bump against one another before stopping.

Still, these imperfections could provide insights into how natural swarms of animals work, says L. Mahadevan, a mathematician and physicist at Harvard who is not connected to the work. He imagines using swarms of Kilobots to test how collective behaviors in insects develop, compete and evolve. Even questions such as how much of human behaviour is determined by centralized thought versus localized reflexes could be informed by Kilobot experiments. "It's only limited by your imagination, I think," he says. "It's going to be a fantastic toolkit."

Nature | doi:10.1038/nature.2014.15714

\section{References}

1. Rubenstein, M., Cornejo, A. \& Nagpal, R. Science 345, 795-799 (2014).

2. Yim, M., Zhang, Y., Roufas, K., Duff, D. \& Eldershaw, C. Mechatronics 7, 442-451 (2002). 